\title{
Wigglesworthia gen. nov. and Wigglesworthia glossinidia sp. nov., Taxa Consisting of the Mycetocyte-Associated, Primary Endosymbionts of Tsetse Flies
}

\author{
SERAP AKSOY \\ Department of Internal Medicine, Yale University School of Medicine, \\ New Haven, Connecticut 06510
}

\begin{abstract}
The primary endosymbionts (P-endosymbionts) of tsetse flies (Diptera: Glossinidae) are harbored inside specialized cells (mycetocytes) in the anterior region of the gut, and these specialized cells form a white, U-shaped organelle called mycetome. The P-endosymbionts of five tsetse fly species belonging to the Glossinidae have been characterized morphologically, and their $16 \mathrm{~S}$ ribosomal DNA sequences have been determined for phylogenetic analysis. These organisms were found to belong to a distinct lineage related to the family Enterobacteriaceae in the $\gamma$ subdivision of Proteobacteria, which includes the secondary endosymbionts of various insects and Escherichia coli. These bacteria are also related to the P-endosymbionts of aphids, Buchnera aphidicola. Signature sequences in the $16 \mathrm{~S}$ ribosomal DNA and genomic organizational differences which distinguish the tsetse fly P-endosymbionts from members of the Enterobacteriaceae and from the genus Buchnera are described in this paper. I propose that the P-endosymbionts of tsetse flies should be classified in a new genus, the genus Wigglesworthia, and a new species, Wigglesworthia glossinidia. The P-endosymbiont found in the mycetocytes of Glossina morsitans morsitans is designated the type strain of this species.
\end{abstract}

Many insects that have single food sources have developed obligatory associations with prokaryotic microorganisms $(7,9)$. All members of the family Glossinidae harbor primary endosymbionts (P-endosymbionts) in specialized organelles called mycetomes, which consist of cells (mycetocytes) that contain these microorganisms (7). In members of the genus Glossina, the mycetomes are white, U-shaped organelles that are localized in the anterior region of the gut. In addition to their mycetome-associated P-endosymbionts, many Glossina species carry secondary endosymbionts (S-endosymbionts) that are associated with their midgut cells (28). In 1907 Stuhlman found bacteria in the intestines of tsetse flies (31), and in 1919 Rouboud described a mycetome-like organelle that he observed in the tsetse fly midgut (26), an observation which was confirmed by Wigglesworth in 1929 (35) and by Buxton in 1955 (8). In 1972, Reinhard et al. described the results of ultrastructural studies of large bacteroids (length, up to $8 \mu \mathrm{m}$ ) which they found in tsetse fly mycetomes (25). In 1974 Huebner and Davey reported that bacteroids are present in tsetse fly ovary tissue (11), and Pinnock and Hess distinguished the mycetome organisms from the smaller endosymbionts found in midgut cells (24).

The endosymbionts are essential for the survival and wellbeing of their hosts in many cases $(7,9)$. In tsetse flies, experimental elimination of these organisms by prokaryote-specific antibiotic treatment $(10,24,30)$ and lysozyme treatment (19) or by treatment with antibodies specifically raised against the endosymbionts $(20,21)$ results in retarded growth of the insect and a decrease in egg production, which in turn results in a loss of the ability of the aposymbiotic hosts to reproduce. The ability to reproduce can be partially restored if the aposymbiotic tsetse flies receive a bloodmeal that is supplemented with B-complex vitamins (thiamine, pantothenic acid, pyridoxine, folic acid, and biotin), suggesting that the endosymbionts probably play a role in metabolism that involves these compounds (22). The individual functions of the P- and S-endosymbionts are not known. It is thought that the tsetse fly gut organisms are maternally transmitted to intrauterine larvae via milk secretions of the mother (13).

Recent advances in molecular techniques, as well as the use of nucleic acid sequences in phylogenetic reconstructions, have provided additional insight into the relationships among bacteria (37). The phylogenetic positions of the three distinct endosymbionts found in tsetse flies have been determined by a 16S ribosomal DNA (rDNA) sequence analysis of the P- and S-endosymbionts associated with gut tissues and the microorganisms found in the ovaries $(1,3,23)$. A phylogenetic analysis of the tsetse fly S-endosymbiont 16S rDNA sequence has shown that this organism is a member of the family Enterobacteriaceae in the $\gamma-3$ subdivision of the Proteobacteria (3). In vitro cell-free cultures of these $\mathrm{S}$-endosymbionts have also been established $(3,34)$. The microorganisms harbored in tsetse fly ovaries have also been characterized phylogenetically and have been found to belong to the genus Wolbachia in the $\alpha$ subdivision of the Proteobacteria (23). The P-endosymbionts of tsetse flies were isolated from mycetomes of five species of flies belonging to the three subgenera of the genus Glossina (1). A preliminary analysis of the $16 \mathrm{~S}$ rDNA-encoding sequences of these organisms revealed that they are different from the S-endosymbionts and the bacteria that reside in ovaries and instead represent a distinct lineage in the $\gamma$ subdivision of the Proteobacteria (1). Similarly, a 16S rDNA sequence comparison revealed that the P-endosymbionts of 12 different species of aphids belong to a distinct lineage in the $\gamma$ subdivision of the Proteobacteria $(15,16,32)$. These endosymbionts have been placed in the genus Buchnera, and Buchnera aphidicola, the endosymbiont of Schizaphis graminum, has been designated the type species of this genus (16).

The intracellular P-endosymbionts of tsetse flies have not been successfully cultivated in vitro or in tissue culture yet, but according to Rule 16a of the International Code of Nomenclature of Bacteria, in cases where a species has not been maintained in laboratory culture, a description or preserved specimen may serve as the type strain $(12,36)$. Thus, the obligate symbionts of cockroaches, the spirochetes found in 


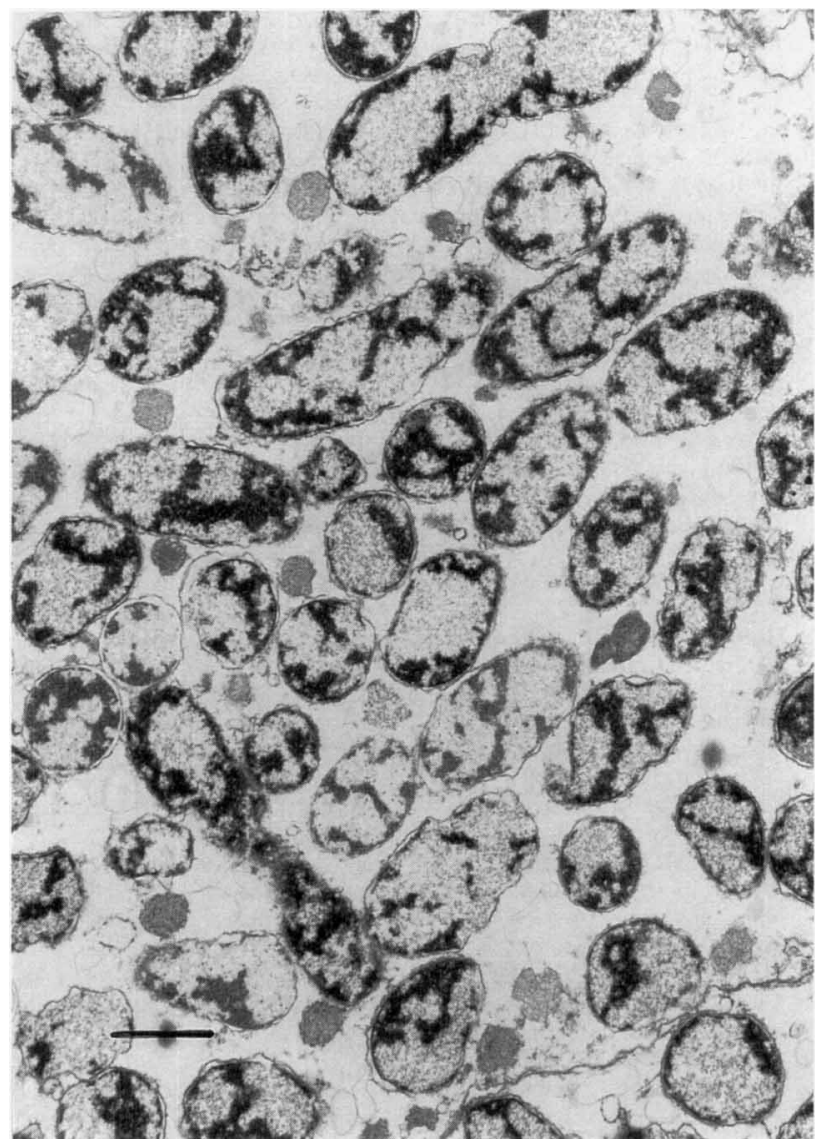

FIG. 1. Electron micrograph of $W$. glossinidia in a mycetome of $G$. morsitans The endosymbionts are surrounded by a two-layer membrane. Bar $=2 \mu \mathrm{m}$.

woodeating cockroaches and termites, have been assigned to new genera and species (7). Similarly, it has not been possible to cultivate members of the genus Wolbachia which cause reproductive incompatibility in culture (33). Recently, the International Committee on Systematic Bacteriology has proposed a new prokaryotic category, the category Candidatus, for characterization of organisms that are difficult to cultivate (17). Clearly, Candidatus status instead of formal recognition of a taxon could lead to contentious discussions. In this paper, I describe a new genus, the genus Wigglesworthia (Wigg.les. worth'i.a. M.L. fem. n. Wigglesworthia, named after the parasitologist V. B. Wigglesworth), and a new species, Wigglesworthia glossinidia (glos.si.nid i.a. M.L. fem. adj. glossinidia, referring to Glossina, the genus of tsetse flies) for the P-endosymbionts of Glossina species. The P-endosymbiont of Glossina morsitans morsitans is designated the type strain of this species. In addition to morphological characteristics, the 1,100bp $16 \mathrm{~S}$ rDNA sequences of the P-endosymbionts of five distantly related Glossina species were determined and phylogenetically analyzed (1). Signature sequences in the $16 \mathrm{~S}$ rDNAs that distinguish these organisms from members of the family Enterobacteriaceae and the genus Buchnera and the genomic organization of the rDNA locus are discussed below.

Description of Wigglesworthia gen. nov. Mycetomes are packed with rod-shaped microorganisms that are up to 4 to 5 $\mu \mathrm{m}$ long (Fig. 1). The microorganisms are in the cytoplasm of the specialized epithelial cells called mycetocytes. They have a typical gram-negative cell wall and lack flagella. The cells appear to divide by binary fission. These organisms have not been cultivated outside their hosts, although it is possible to keep them metabolically active for at least $72 \mathrm{~h}$ under transient tissue culture conditions (2). It is possible to study the gene expression and protein transport in P-endosymbionts of tsetse flies by using transient tissue culture conditions, and in this way the major protein product has been identified as a groEL homolog by immunological cross-reactivity (2).

On the basis of their 16S rDNA sequences, members of this genus form a distinct lineage in the $\gamma-3$ subdivision of the Proteobacteria. As shown in the phylogenetic tree in Fig. 2, they are closely related to members of the genus Buchnera, to Sendosymbionts of other insects, and to Escherichia coli. Table 1 shows the sequence signatures in the 16S rDNA that distinguish the genus Wigglesworthia from members of the genus Buchnera and from other members of the $\gamma-3$ subgroup.

Description of Wigglesworthia glossinidia sp. nov. The morphological description is the same as that given above for the genus. The type strain is the primary endosymbiont of $G$. morsitans morsitans.

$W$. glossinidia $16 \mathrm{~S}$ rDNA sequences have also been obtained from the following species and subspecies of tsetse flies: Glossina tachinoides, Glossina palpalis palpalis, Glossina austeni, and Glossina brevipalpis. These sequences were analyzed phylogenetically by performing a parsimony analysis (Fig. 2).

Additional information for differentiating $W$. glossinidia from other symbionts. The S-endosymbionts of tsetse flies harbored in midgut cells and the Wolbachia-like bacteria of tsetse flies harbored in ovary tissues are morphologically different from members of the genus Wigglesworthia. They are short rods (length, 1 to $2 \mu \mathrm{m}$ ) and are inside cells surrounded by host membranes (25). There are clear lytic zones surrounding the cells that are reminiscent of the zones found with members of the family Rickettsiaceae, to which the genus Wolbachia is related $(24,29)$. Since a phylogenetic analysis of the S-endosymbionts from tsetse flies showed that these organisms are not related to rickettsiae, it is not clear what the clear lytic zones mean.

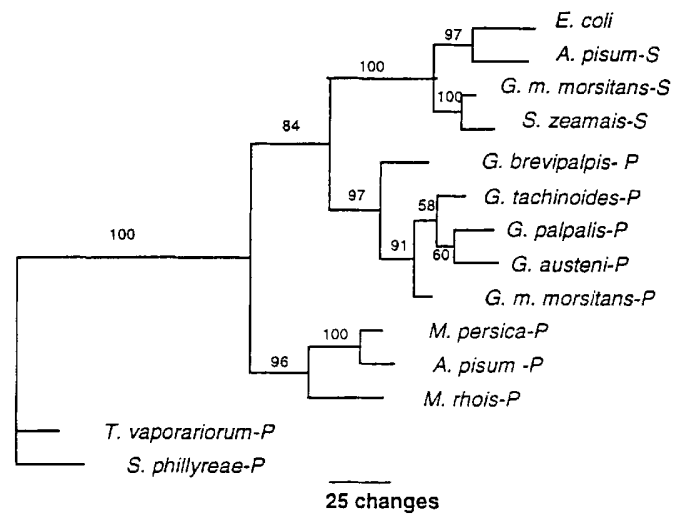

FIG. 2. Phylogenetic tree based on the 16S rDNA sequences of the endosymbionts of aphids, tsetse flies, and whiteflies and E. coli. -P, primary mycetocyte endosymbiont; -S, secondary endosymbiont. All of the names except $E$. col are the names of the insect species that contain the endosymbionts. Abbreviations: E. coli, Escherichia coli; A. pisum, Acyrthosiphon pisum; G. m. morsitans, Glossina morsitans morsitans; $S$. zeamais, Sitophilus zeamais; $G$. brevipalpis, Glossina brevipalpis; $G$. tachinoides, Glossina tachinoides; $G$, palpalis, Glossina palpalis; G. austeni, Glossina austeni; M. persica, Myzus persica; M. rhois, Melaphis rhois; T. vaporarionum, Trialeurodes vaporariorum; S. phillyreae, Siphoninus phillyreae. This tree is the majority-rule consensus tree obtained after 100 bootstrap runs; the numbers are bootstrap indices for the levels of support of the nodes. There were 220 informative characters. Reprinted from Insect Molecular Biology (2) with permission of the publisher. 
TABLE 1. Sequence signatures that distinguish $W$. glossinidia from B. aphidicola and other members of the $\gamma-3$ subgroup

\begin{tabular}{lccc}
\hline \multirow{2}{*}{ Position $^{a}$} & \multicolumn{3}{c}{ Nucleoside in: } \\
\cline { 2 - 4 } & Nigglesworthia $^{b}$ & Buchnera $^{c}$ & $\gamma-3$ subgroup $^{d}$ \\
\hline 75 & $\mathrm{~T}$ & $\mathrm{~A}$ & $\mathrm{G}$ \\
79 & $\mathrm{~T}$ & $\mathrm{~A}$ & $\mathrm{G}$ \\
188 & $\mathrm{~T}$ & $\mathrm{~A}$ & $\mathrm{C}$ \\
210 & $\mathrm{~A}$ & $\mathrm{~T}$ & $\mathrm{C}$ \\
225 & $\mathrm{G}$ & $\mathrm{T}$ & $\mathrm{C}$ \\
592 & $\mathrm{~A}$ & $\mathrm{~T}$ & $\mathrm{G}$ \\
621 & $\mathrm{~A}$ & $\mathrm{C}$ & $\mathrm{G}$ \\
911 & $\mathrm{G}$ & $\mathrm{T}$ & $\mathrm{C}$ \\
\hline
\end{tabular}

${ }^{a}$ The numbers refer to positions in the E. coli sequence (37).

${ }^{b}$ Sequences of the P-endosymbionts obtained from $G$. morsitans morsitans, $G$. tachinoides, and $G$. brevipalpis.

${ }^{c}$ Sequences of the P-endosymbionts ( $B$. aphidicola) obtained from the aphids Myzus persicae, Acyrthosiphon pisum, and Melaphis rhois.

${ }^{d}$ Sequences of $E$. coli, Proteus vulgaris, and the S-endosymbionts of Acrythosiphon pisum, Sitophilus zeamais, and G. morsitans morsitans (18).

The organization and copy number of the 16S rDNA of the genus Wigglesworthia are also different from the organization and copy number of the 16S rDNA operons of Buchnera species and $E$. coli. The free-living bacterium $E$. coli $(4,5)$ and S-endosymbionts of tsetse flies (2) have multiple copies of rDNA operons encoding their $16 \mathrm{~S}$ and 23S rDNA gene products. Members of the genus Wigglesworthia have single operons encoding their 16S rDNA sequences (2), like members of the genus Buchnera (32). While the copy number of the Wigglesworthia rDNA operon is similar to the copy number of the Buchnera rDNA operon, the genomic organization of the $16 \mathrm{~S}$ and 23S rDNA genes in the genus Wigglesworthia is different. In the genus Buchnera, these genes are not genetically linked $(14,27)$, while in the genus Wigglesworthia they are encoded by the same operon (i.e., they are transcriptionally linked) (2). In terms of genetic organization, this is similar to the arrangement found in E. coli (6) and in the tsetse fly S-endosymbiont genome (unpublished data) (1).

Nucleotide sequence accession numbers. The GenBank accession numbers for the $16 \mathrm{~S}$ rDNA sequences used to construct the phylogenetic tree are as follows: E. coli, V00348; S-endosymbiont of Acyrthosiphon pisum, M27040; S-endosymbiont of $G$. morsitans morsitans, M99060; S-endosymbiont of Sitophilus zeamais, M85269; P-endosymbiont of G. brevipalpis, L37341; P-endosymbiont of G. tachinoides, L37342; P-endosymbiont of $G$. palpalis, L37343; P-endosymbiont of G. austeni, L37340; P-endosymbiont of G. morsitans morsitans, L37339; P-endosymbiont of Myzus persica, M63249; P-endosymbiont of A. pisum, M27049; P-endosymbiont of Melaphis rhois, M63255; P-endosymbiont of Trialeurodes vaporarionum, Z11928; and Pendosymbiont of Siphoninus phillyreae, Z11927.

I am grateful to Paul Baumann for his critical reading of the manuscript.

This research was supported in part by grant AI-28778 from the National Institutes of Health Tropical Diseases Research Unit and by grant NIH-AI-34033.

\section{REFERENCES}

1. Aksoy, S. 1995. Molecular analysis of the endosymbionts of tsetse fly; $16 \mathrm{~S}$ rDNA locus and over-expression of molecular chaperonins. Insect. Mol. Biol. 4:23-29

2. Aksoy, S., A. Pourhosseini, and A. Chow. 1995. Mycetome endosymbionts of tsetse flies constitute a distinct lineage related to Enterobacteriaceae. Insect. Mol. Biol. 4:15-22.
3. Beard, C. B., S. L. O'Neill, P. W. Mason, L. Mandelco, C. R. Woese, R. B. Tesh, F. F. Richards, and S. Aksoy. 1993. Genetic transformation and phylogeny of bacterial symbionts from tsetse. Insect Mol. Biol. 1:123131

4. Boros, I., A. Kiss, and P. Vetetianer. 1979. Physical map of the seven ribosomal RNA genes of Escherichia coli. Nucleic Acids Res. 6:1817-1830.

5. Brosius, J., T. J. Dull, D. D. Sleeter, and H. Noller. 1981. Gene organization and primary structure of a ribosomal operon from Escherichia coli. J. Mol. Biol. 148:107-127.

6. Brosius, J., A. Ullrich, M. A. Raker, A. Gray, T. J. Dull, R. R. Gutell, and H. F. Noller. 1981. Construction and fine mapping of recombinant plasmids containing the $r m B$ ribosomal RNA operon of $E$. coli. Plasmid 6:112118 .

7. Buchner, P. 1965. Endosymbiosis of animals with plant microorganisms, p. 210-338. Interscience Publishers, Inc., New York.

8. Buxton, P. A. 1955. A natural history of tsetse flies: an account of the biology of the genus Glossina (Diptera). London School Hyg. Trop. Med. Mem. 10:80-82.

9. Douglas, A. E. 1989. Mycetocyte symbiosis in insects. Biol. Rev. 64:409-434

10. Hill, P. D. S., and J. A. Campbell. 1973. The production of symbiont-free Glossina morsitans and an associated loss of female fertility. Trans. R. Soc. Trop. Med. Hyg. 67:727-728.

11. Huebner, E., and K. G. Davey. 1974. Bacteroids in the ovaries of the tsetse fly. Nature (London) 249:260-261.

12. Lapage, S. P., P. H. A. Sneath, E. F. Lessel, V. B. D. Skerman, H. P. R. Seeliger, and W. A. Clark (ed). 1992. International code of nomenclature of bacteria. 1990 Revision. American Society for Microbiology, Washington, D.C.

13. Ma, W.-C., and D. L. Denlinger. 1974. Secretory discharge and microflora of milk gland in tsetse flies. Nature (London) 247:301-303.

14. Munson, M. A., L. Baumann, and P. Baumann. 1993. Buchnera aphidicola (a prokaryotic endosymbiont of aphids) contains a putative $16 \mathrm{~S}$ rRNA operon unlinked to the 23S rRNA-encoding gene: sequence determination, and promoter and terminator analysis. Gene 137:171-178.

15. Munson, M. A., P. Baumann, M. A. Clark, L. Baumann, N. A. Moran, D. J. Voegtlin, and B. C. Campbell. 1991. Evidence for the establishment of aphid-eubacterium endosymbiosis in an ancestor of four aphid families. J. Bacteriol. 173:6321-6324.

16. Munson, M. A., P. Baumann, and M. G. Kinsey. 1991. Buchnera gen. nov. and Buchnera aphidicola sp. nov., a taxon consisting of the mycetocyteassociated, primary endosymbionts of aphids. Int. J. Syst. Bacteriol. 41:566568.

17. Murray, R. G. E., and E. Stackebrandt. 1995. Taxonomic note: implementation of the provisional status Candidatus for incompletely described procaryotes. Int. J. Syst. Bacteriol. 45:186-187.

18. Neefs, J.-M., Y. Van der Peer, L. Hendricks, and R. De Wachter. 1990. Compilation of small ribosomal subunit RNA sequences. Nucleic Acids Res. 18:2217-2337.

19. Nogge, G. 1976. Sterility in tsetse flies caused by loss of symbionts. Experientia 32:995.

20. Nogge, G. 1978. Aposymbiotic tsetse flies, Glossina morsitans morsitans obtained by feeding on rabbits immunized specifically with symbionts. J. Insect Physiol. 24:299-304.

21. Nogge, G. 1980. Elimination of symbionts of tsetse flies (Glossina m. morsitans Westw.) by help of specific antibodies, p. 445-457. In W. Schwemmler and H. Schenk (ed.), Endocytobiology. W. de Gruyter, Berlin.

22. Nogge, G. 1981. Significance of symbionts for the maintenance of an optimal nutritional state for successful reproduction in hematophagous arthropods. Parasitology 82:299-304.

23. O'Neill, S. L., R. H. Gooding, and S. Aksoy. 1993. Phylogenetically distant symbiotic microorganisms reside in Glossina midgut and ovary tissues. Med. Vet. Entomol. 7:377-383.

24. Pinnock, D. E., and R. T. Hess. 1974. The occurrence of intracellular rickettsia-like organisms in the tsetse flies, Glossina morsitans, G. fuscipes, $G$. brevipalpis and G. pallidipes. Acta Trop. 31:70-79.

25. Reinhardt, C., R. Steiger, and H. Hecker. 1972. Ultrastructural study of the midgut mycetome bacteroides of the tsetse fly Glossina morsitans, G. fuscipes and G. brevipalpis (Diptera: Brachycera). Acta Trop. 29:280-288.

26. Roubaud, B. 1919. Les particularites de la nutrition et de la vie symbiotique chez les mouches tsetse. Ann. Inst. Pasteur 33:489-537.

27. Rouhbakhsh, D., N. A. Moran, L. Baumann, L., D. J. Voegtlin, and P. Baumann. 1994. Detection of Buchnera, the primary prokaryotic endosymbionts of aphids, using the polymerase chain reaction. Insect Mol. Biol. 3:213-217.

28. Schlein, Y. 1976. Lethal effect of tetracycline on tsetse following damage to bacteroid symbionts. Experientia 33:450-451.

29. Shaw, M. K., and S. K. Moloo. 1991. Comparative studies on rickettsia-like organisms in the midgut epithelial cells of different Glossina species. Parasitology 102:193-199.

30. Southwood, T. R. E., S. Khalaf, and R. E. Sinden. 1975. The micro-organisms of tsetse flies. Acta Trop. 32:259-266. 
31. Stuhlman, F. 1907. Beitrage zur Kenntnis der Tsetsefliege. Arb. Gesundh. Amte (Berlin) 26:301-308.

32. Unterman, B. M., P. Baumann, and D. L. McLean. 1989. Pea aphid symbiont relationships established by 16S rRNAs. J. Bacteriol. 171:2970-2974.

33. Weiss, E., G. Dasch, and K. P. Chang. 1984. Genus VII. Wolbachia, p. 711-713. In N. R. Krieg and J. G. Holt (ed.), Bergey's manual of systematic bacteriology, vol. 1. The Williams \& Wilkins Co., Baltimore.

34. Welburn, S. C., D. S. Ellis, and I. Maudlin. 1987. In vitro cultivation of rickettsia-like organisms from Glossina spp. Ann. Trop. Med. Parasitol. 81:331-335.

35. Wigglesworth, V. B. 1929. Digestion in the tsetse fly: a study of the structure and function. Parasitology 21:288-321.

36. Wink, M. 1979. The endosymbionts of Glossina morsitans and G. palpalis: cultivation experiments and some physiological properties. Acta Trop. 36: 215-222.

37. Woese, C. R. 1987. Bacterial evolution. Microbiol. Rev. 51:221-271. 